\title{
Prevalência de cárie dentária em crianças da faixa etária de 0 a 6 anos matriculadas em creches: importância de fatores socioeconômicos*
}

\section{Prevalence of dental caries in preschool children attending nursery: the influence of socioeconomic factors}

\author{
Nilce E. Tomita, Vitoriano T. Bijella, Eymar S. Lopes e Laércio J. Franco \\ Departamento de O dontologia Social da Faculdade de O dontologia de Bauru da Universidade de \\ São Paulo. Bauru, SP - Brasil (N.E.T., V.T.B., E.S.L.), Departamento de Medicina Preventiva da \\ Universidade Federal de São Paulo. São Paulo, SP - Brasil (L.J.F.)
}

\begin{abstract}
Resumo
Foi avaliada a prevalência de cárie na dentição decídua de crianças entre 0 e 6 anos, matriculadas em creches dos Municípios de Bauru e São Paulo, SP (Brasil). O primeiro grupo (Bauru) não recebia cuidados sistematizados de saúde na instituição e o segundo (São Paulo) apresentava uma rotina de cuidados como norma institucional. Foram analisadas as variáveis relativas aos modos de viver desses grupos populacionais e sua associação com a ocorrência de cárie, efetuando um estudo de caso para caracterização de fatores coletivos de risco à cárie. Através de análise de regressão múltipla, verificou-se a influência da idade e frequiência de consultas odontológicas sobre a prevalência de cárie na amostra estudada $(\mathrm{p}<0,05)$. Na faixa etária de $5-6$ anos, $23,3 \%$ das crianças de Bauru e 9,3\% de São Paulo estavam isentas de cárie, contra a expectativa de 50\% prevista na Meta n.o 1 da Organização Mundial da Saúde para o ano 2000. A prevalência de cárie foi mais elevada em Bauru nas crianças de 3-4 e 5-6 anos, apresentando significância estatística apenas para o grupo 3-4 anos $(\mathrm{p}<0,05)$. Não foram observadas diferenças estatisticamente significantes entre os sexos quanto à ocorrência de cárie.
\end{abstract}

Cárie dentária, epidemiologia. Levantamentos de saúde bucal. Fatores de risco.

\begin{abstract}
s
Oral health condition in samples of children from zero to six years old, examined in day nurseries for children from Bauru and S. Paulo County (Brazil) by the caries indicator in primary dentition - $d m f s$, is assessed. The first group did not receive oral health care where any as the second group received the standard oral care provided by the institution. Variables related to way of life
\end{abstract}

\footnotetext{
* Baseado na dissertação de mestrado do curso de Pós-Graduação em Odontopediatria da Faculdade de Odontologia de Bauru-USP, 1993. Apresentado na XI Reunião Anual da Sociedade Brasileira de Pesquisas Odontológicas/Divisão Brasileira da IADR (International Association for Dental Research), Águas de São Pedro-SP, 4 a 7/setembro/1994.

Correspondência para/Correspondence to: Nilce E. Tomita - Departamento de Odontologia Social da Faculdade de Odontologia de Bauru da Universidade de São Paulo. Al. Otávio Pinheiro Brisolla, 9-75 - 17043-101 Bauru, SP - Brasil. Fax: (014) 223.5525 E-mail: netomita@usp.br Edição subvencionada pela FAPESP. Processo 95/2290-6.

Recebido em 5.12.1994. Reapresentado em 3.8.1995. Aprovado em 13.3.1996.
} 
and their relationship to the presence of caries were evaluated. Multiple regression analysis showed a statistically significant association of age and frequency of dental visits with the prevalence of dental caries $(p<0.05)$. The data also showed that $23.3 \%$ of the children from Bauru, and $9.3 \%$ of those from S. Paulo were free of caries, numbers that are very far from the 50\% proposed by WHO for the year 2000. The overall prevalence of dental caries was greater for the Bauru group than for the S. Paulo group; however, statistical significance was only found in the 3-4 age-group $(p<0.05)$. Sex differences in the occurrence of dental caries were not statistically significant.

Dental caries, epidemiology. Dental health surveys. Risk factors.

\section{INTRODUÇÃO}

A saúde bucal da população brasileira, expressa pelos índices de cárie, retrata níveis de alta precariedade. Levantamento nacional, realizado pelo Ministério da Saúde, em 1986, registrou CPOD de 6,65 aos 12 anos de idade, considerado um dos mais elevados do mundo ${ }^{8}$.

A prevalência de doenças bucais, como a cárie dentária, é influenciada por outros fatores, além dos etiológicos já conhecidos (a tríade de KEYES: hospedeiro, microbiota e dieta, modulados pelo fator tempo), como a renda familiar. O levantamento epidemiológico realizado no Brasil, em 1986, evidenciou que a criança pertencente à família que recebe até dois salários-mínimos mensais tem $43,8 \%$ dos dentes com lesão em seu registro de história de cárie, ao passo que aquela pertencente à família com renda mensal acima de 5 salários-mínimos apresenta $22,4 \%$ dos dentes cariados. Também a prevenção de cárie dentária parece ser menos eficaz nas faixas de renda mais baixas: $12,5 \%$ das crianças entre 6 e 12 anos apresentam-se livres de cárie no primeiro grupo (menos de dois salários-mínimos), em comparação aos 18,2\% livres de cárie no segundo grupo, ou seja, aproximadamente $50 \%$ maior $^{7,8,34}$.

Levando-se em conta que metade dos 59 milhões de brasileiros entre 0 e 17 anos pertence a famílias cuja faixa de renda mensal per capita não ultrapassa meio salário-mínimo, que $60 \%$ dos recém-nascidos vivem em moradias sem água tratada e rede de esgoto, e que cerca de oito milhões de crianças na faixa etária de 7 a 17 anos deixaram a escola, trocando-a pelo ingresso precoce no mercado de trabalho ou vivendo na linha da marginalidade ${ }^{2,15}$, não é de se estranhar que os níveis de morbidade infantil alcancem altos patamares, ou que doenças preveníveis, como a cárie dentária e a doença periodontal, apresentem perfil de elevada endemicidade.
As condições de saúde bucal do pré-escolar não têm sido documentadas na mesma extensão que a saúde bucal do escolar. Isto provavelmente ocorre porque a dentição decídua, com freqüência, não é considerada tão importante quanto a permanente. Em muitos países, o ingresso na escola dá-se após os 6 anos de idade, o que explica serem os levantamentos epidemiológicos mais freqüentemente realizados, focalizando as crianças em idade escolar, pois são de mais fácil localização e identificação ${ }^{19}$.

É conhecida, no entanto, a relação entre história de cárie na dentição decídua e seus níveis na dentição permanente ${ }^{17,23,24,32,38}$ devido à transmissibilidade da doença ${ }^{9,37}$.

É sabido que quanto mais precocemente ocorrer a infecção na dentição decídua da criança, por microrganismos patogênicos, maior a prevalência de cárie nessa dentição ${ }^{1,14}$. Hábitos arraigados no cotidiano do cuidado infantil, como provar os alimentos com a colher da criança, ou resfriá-los soprando, são as principais formas de transmissão da infecção da mãe para o filho ${ }^{1}$.

A melhoria do cuidado durante os anos pré-escolares reduziria a necessidade de restaurações ou extrações ${ }^{44}$, uma vez que a cárie é considerada uma doença da infância ${ }^{12}$.

Além disso, a avaliação de fatores sociais e biológicos, que interferem no desenvolvimento de doenças bucais, pode significar contribuição a estudos populacionais que objetivem a identificação do público-alvo preferencial a receber atenção no âmbito dos serviços públicos, tendo em vista a escassa destinação de recursos ao setor saúde e a amplidão das necessidades acumuladas.

A comparação da prevalência de cárie entre crianças de creches, onde não há cuidados de saúde bucal instituídos, com aquelas de creches cuja rotina contempla um plano de cuidados, pode fornecer informações acerca do perfil de saúde bucal de crianças nessa faixa etária, de acordo com a rotina adotada e as características socioeconômicas desses grupos. 
Os principais objetivos do presente estudo, focalizando crianças da faixa etária de 0 a 6 anos, são:

a) Avaliar a prevalência de cárie na dentição decídua em dois grupos de crianças, na faixa etária de 0 a 6 anos, com características sociais distintas.

b) Avaliar algumas variáveis sociais e sua influência sobre a ocorrência de cárie na dentição decídua.

c) Avaliar o acesso à assistência odontológica e sua repercussão no quadro de saúde bucal da amostra estudada.

\section{CASUÍSTICA E MÉTODO}

O conjunto de crianças estudadas foi formado por dois grupos: crianças de creches de São Paulo e crianças de creches de Bauru.

Os grupos foram formados em função da presença das crianças quando da visita dos examinadores às instituições, sendo contemplada a totalidade das crianças que se encontravam na creche nessa ocasião.

O grupo de São Paulo foi constituído por crianças de duas creches públicas, cuja proposta pedagógica baseiase na linha interacional-construtivista. O grupo de Bauru foi formado por crianças de nove creches e berçários públicos e privados da cidade.

A coleta de dados foi estruturada em duas etapas: na avaliação da história de cárie nas 699 crianças que compuseram a amostra (Figura 1); e na aplicação de um questionário de saúde junto aos pais de crianças de uma sub-amostra (Figura 2), no período de setembro a novembro de 1991.

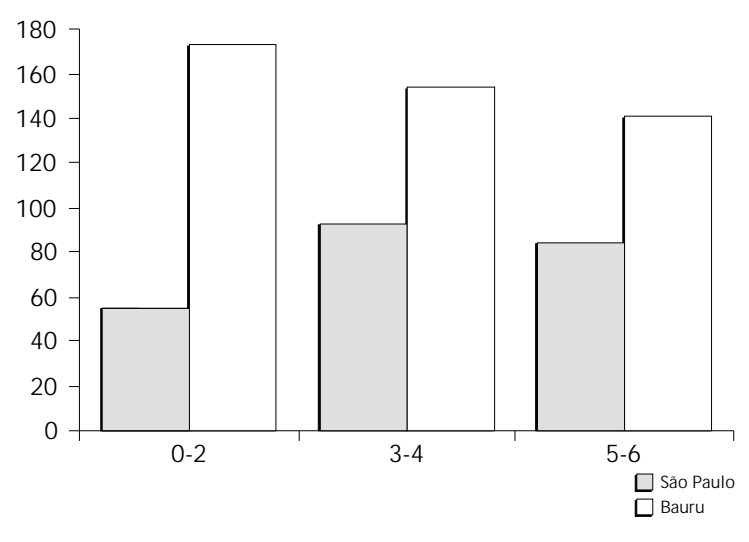

Figura 1 - Distribuição da amostra segundo a procedência, por faixa etária, nos M unicípios de Bauru e São Paulo.

Na primeira etapa, a avaliação clínica da história de cárie deu-se mediante inspeção visual da cavidade bucal das crianças, com auxílio de espelho clínico e, quando necessário, sonda exploradora ${ }^{26}$. Foi utilizado o índice ceos $\left(\right.$ Gruebbel $\left.^{18}\right)$, que possibilita a expressão quantitativa da prevalência de cárie na dentição decídua. Sua base conceitual consiste na determinação da história de cárie, passada ou presente, através da experiência individual da

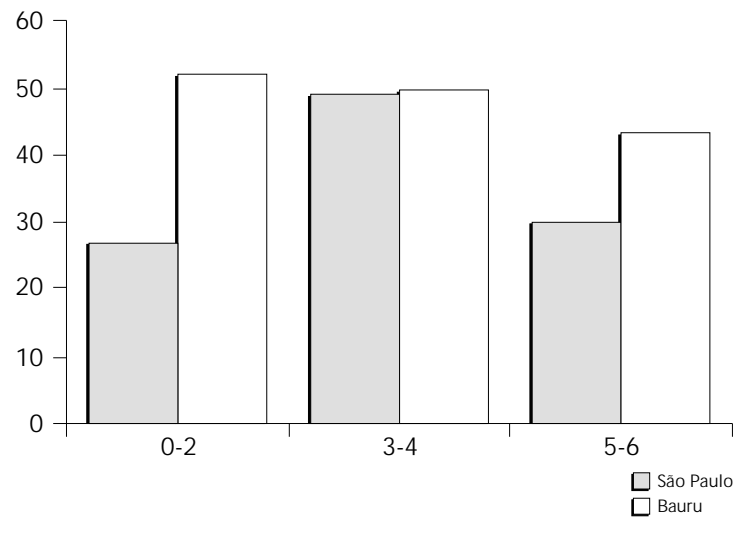

Figura 2 - Amostra examinada segundo a realização de entrevista, por faixa etária, nos municípios de Bauru e São Paulo.

doença, expressa pelo número de superfícies que apresentam, no momento do exame, lesões de cárie ou restaurações, ou quando da perda decorrente de extração do elemento dentário motivada por cárie ${ }^{11,29}$.

A segunda etapa da coleta de dados consistiu de entrevistas realizadas em uma subamostra de crianças examinadas, junto aos pais/responsáveis, à entrada ou saída da instituição. O questionário foi constituído por uma série de quesitos ordenados em forma de perguntas abertas e fechadas, com abordagem de assuntos como identificação, história reprodutiva da mãe, situação socioeconômica, condições de moradia, hábitos alimentares, aleitamento materno, condições de saúde, acesso a serviços de saúde, medidas de cuidado em saúde bucal, escolaridade materna e paterna, ocupação da mãe e da pessoa de maior renda na família.

Os dados analisados são referentes a 699 fichas de exame da história de cárie e a 251 questionários referentes às entrevistas com os pais das crianças de São Paulo e Bauru. Os dados foram processados através do software DBase III Plus (ASHTON-TATE), após o agrupamento de algumas variáveis.

Para a análise dos resultados foram utilizados os testes de Qui-Quadrado e t de "Student". Para análise simultânea do conjunto de variáveis socioeconômicas e sua influência sobre a experiência de cárie nos grupos estudados foram utilizados testes de regressão múltipla e análise de correlação .

Fixou-se em 0,05 o nível para rejeição da hipótese de nulidade, assinalando-se com um asterisco os valores significantes na apresentação das tabelas. Nas comparações onde o nível de rejeição foi menor, o valor foi especificado.

\section{RESULTADOS E DISCUSSÃO}

As semelhanças entre os dois grupos podem ser verificadas por características como estrutura etária (Figuras 1 e 2), média de idade em anos (Tabela 1) e número médio de dentes presentes por faixa etária (Figura 3). 
Tabela 1 - Média e desvio-padrão (DP) da idade para as crianças com entrevistas, nos Municípios de Bauru e São Paulo.

\begin{tabular}{lccc}
\hline Procedência & \multicolumn{3}{c}{ Idade (anos) } \\
& Média & D P & N \\
\hline Bauru & 3,3 & 1,5 & 145 \\
São Paulo & 3,6 & 1,7 & 106 \\
\hline n.s. & & &
\end{tabular}

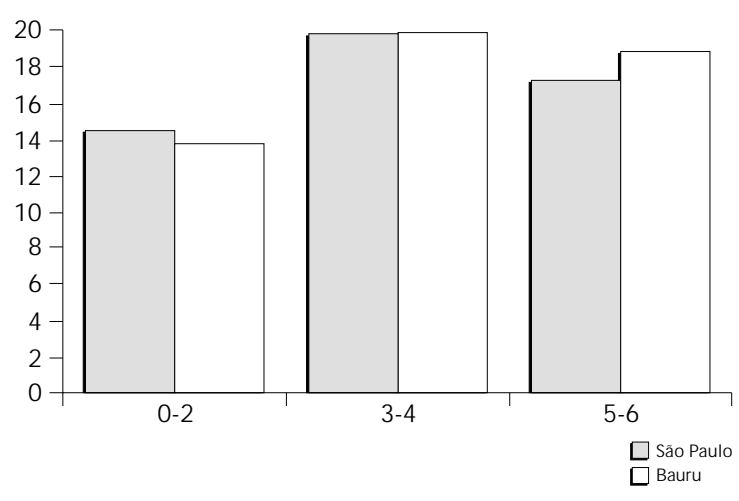

Figura 3 - Número médio de dentes presentes nas crianças de Bauru e São Paulo, por faixa etária.

As crianças do grupo de São Paulo eram provenientes de várias localidades do município, onde as águas de abastecimento público são fluoretadas desde 1985. No Município de Bauru, cerca de $90 \%$ da população recebe água tratada e fluoretada. Devido à dificuldade em obter dados quanto ao percentual de crianças beneficiadas por essa medida sanitária coletiva, esta variável não pôde ser avaliada entre os dois grupos.

A avaliação de indicadores socioeconômicos apresenta grande importância em estudos de saúde infantil, pois permite descrever a situação dessa po- pulação e conhecer as suas condições de vida, moradia e saneamento. A renda familiar, um dos indicadores que permite avaliar a situação socioeconômica de uma população, é considerada uma variável com alto poder discriminatório, isto é, crianças pertencentes a famílias com diferentes níveis de renda apresentam diferenças importantes nos indicadores de saúde ${ }^{4,25,42}$.

$\mathrm{Na}$ Tabela 2, podem-se observar diferenças significantes na composição da amostra, com maior percentual de crianças, no grupo de São Paulo, na faixa de renda superior a 5 salários-mínimos mensais e ampla predominância, no grupo de Bauru, das faixas de renda até 4 salários-mínimos. Embora a variável renda apresente limitações quando se comparam grupos com inserções sociais distintas ${ }^{3,16}$, a mesma evidencia uma associação negativa com a ocorrência de cárie cin $^{13,30,33,35,45}$.

A meta 1 da $\mathrm{OMS}^{22}$ prevê que $50 \%$ das crianças entre 5 e 6 anos de idade devem estar livres de cárie no ano 2000. Na Tabela 3, podem ser observados os números e percentuais de crianças isentas de cárie, por faixa etária. Nota-se que, com exceção da faixa etária de 0-2 anos, houve maior proporção de crianças livres de cárie no grupo de São Paulo. No entanto, no conjunto da amostra, apenas no grupo etário de 3-4 anos a diferença apresentou significância estatística.

Na Tabela 4 pode ser observada a comparação entre o índice ceos nos grupos de Bauru e São Paulo. Em todas as idades, o índice ceos foi maior nas crianças do grupo de Bauru, com significância estatística nas faixas etárias de 3-4 e 5-6 anos $(\mathrm{p} \leq 0,001)$. Os padrões de ocorrência encontram-se de acordo com a literatura, quanto à progressão conforme a idade ${ }^{5,}$ $12,15,30,31,32,38$

Tabela 2 - Distribuição da amostra segundo a renda familiar (em salários-mínimos), por faixa etária, para os grupos de Bauru e São Paulo.

\begin{tabular}{|c|c|c|c|c|c|c|c|c|}
\hline \multirow{3}{*}{$\begin{array}{l}\text { Renda familiar } \\
\text { (salários-mínimos) }\end{array}$} & \multicolumn{6}{|c|}{ Faixa etária (anos) } & & \\
\hline & \multicolumn{2}{|c|}{$0-2$} & \multicolumn{2}{|c|}{$3-4$} & \multicolumn{2}{|c|}{$5-6$} & \multicolumn{2}{|c|}{ Total } \\
\hline & $\mathrm{N}$ & $\%$ & $\mathrm{~N}$ & $\%$ & $\mathrm{~N}$ & $\%$ & $\mathrm{~N}$ & $\%$ \\
\hline \multicolumn{9}{|l|}{ Bauru } \\
\hline $0-2$ & 17 & 32,7 & 11 & 22,0 & 11 & 25,6 & $39 *$ & 26,9 \\
\hline $3-4$ & 21 & 40,4 & 25 & 50,0 & 24 & 55,8 & $70 *$ & 48,3 \\
\hline$\geq 5$ & 14 & 27,0 & 14 & 28,0 & 08 & 18,6 & $36 *$ & 24,9 \\
\hline Total & 52 & 100,0 & 50 & 100,0 & 43 & 100,0 & $145^{*}$ & 100,0 \\
\hline \multicolumn{9}{|l|}{ São Paulo } \\
\hline $0-2$ & 03 & 11,1 & 03 & 6,1 & 0 & 0 & 06* & 5,7 \\
\hline $3-4$ & 11 & 40,7 & 18 & 36,7 & 14 & 46,7 & $43^{*}$ & 40,6 \\
\hline$\geq 5$ & 13 & 48,1 & 28 & 57,1 & 16 & 53,3 & $57 *$ & 53,8 \\
\hline Total & 27 & 100,0 & 49 & 100,0 & 30 & 100,0 & $106 *$ & 100,0 \\
\hline
\end{tabular}

$X^{2}$ calc $=30,06$

$* p<0,0001$ 
Tabela 3 - Percentual de crianças livres de cárie, nos grupos de Bauru e São Paulo, por faixa etária.

\begin{tabular}{|c|c|c|c|c|c|c|}
\hline \multirow[t]{3}{*}{ Procedência } & \multicolumn{6}{|c|}{ Crianças livres de cárie } \\
\hline & \multicolumn{2}{|c|}{$0-2$} & \multicolumn{2}{|c|}{$3-4$} & \multicolumn{2}{|c|}{$5-6$} \\
\hline & $\mathrm{N}$ & $\%$ & $\mathrm{~N}$ & $\%$ & $\mathrm{~N}$ & $\%$ \\
\hline \multicolumn{7}{|l|}{ Bauru } \\
\hline & $46 / 52$ & 88,5 & $13 / 50$ & 26,0 & $4 / 43$ & 9,3 \\
\hline \multicolumn{7}{|l|}{ São Paulo } \\
\hline & $23 / 27$ & 85,2 & $29 / 49$ & 59,2 & $7 / 30$ & 23,3 \\
\hline $\mathrm{X}^{2}$ calc. & & $0,17^{b}$ & & $11,16^{* a}$ & & $2,72^{b}$ \\
\hline
\end{tabular}

Tabela 4 - Índice ceos entre grupos segundo a procedência.

\begin{tabular}{ccccccc}
\hline \multicolumn{2}{l}{ Faixa etária } & \multicolumn{3}{c}{ Bauru } & \multicolumn{4}{c}{ São Paulo } & t & Probabilidade \\
(anos) & ceos & (N) & ceos & (N) & & \\
\hline $0-2$ & 1,18 & $(173)$ & 0,43 & $(54)$ & $-1,242$ & 0,215 \\
$3-4$ & 5,49 & $(151)$ & 2,02 & $(93)$ & $-3,579$ & $0,000^{*}$ \\
$5-6$ & 8,83 & $(141)$ & 4,77 & $(84)$ & $-3,469$ & $0,001^{*}$ \\
\hline * $<<0,05$ & & & & & &
\end{tabular}

Tabela 5 - Índice ceos e número médio de dentes segundo 0 sexo.

\begin{tabular}{lcccccc}
\hline Sexo & \multicolumn{2}{c}{ Masculino } & \multicolumn{2}{c}{ Feminino } & t & Probabilidade \\
& ceos & $(\mathrm{N})$ & $\operatorname{ceos}$ & $(\mathrm{N})$ & & \\
\hline ceos & 3,35 & $(137)$ & 5,01 & $(113)$ & 1,75 & 0,080 \\
No médio & 17,24 & $(137)$ & 18,00 & $(113)$ & 1,36 & 0,175 \\
de dentes & & & & & & \\
\hline
\end{tabular}

A experiência de cárie e o número médio de dentes da subamostra estudada são apresentados na Tabela 5. Não foram observadas diferenças significantes quanto ao sexo em crianças na faixa pré-escolar, em concordância com a literatura ${ }^{11,20,21,32,36,37,40,45}$.

A utilização do coeficiente de correlação parcial, descrito na Tabela 6 , permite a avaliação em conjunto da variação de todos os fatores simultaneamente. A análise da correlação parcial entre as variáveis, tomando por variável dependente o ceos, teve por objetivo avaliar a associação existente quando se elimina a influência das demais variáveis. Sob essa análise, as variáveis que apresentaram valores significantes de influência sobre a experiência de cárie no presente estudo foram a idade e a freqüência de consultas odontológicas.

A análise de regressão múltipla permite uma avaliação global das variáveis inseridas no conjunto de suas inter-relações, uma vez que, a não ser de modo empírico, não se pode confirmar a atuação isolada de qualquer das variáveis na determinação da freqüência de cárie nos grupos estudados. Esse processamen-
Tabela 6 - Coeficientes de correlação entre o índice ceos e algumas variáveis socioeconômicas.

\begin{tabular}{lcc}
\hline Variável & $\begin{array}{c}\text { Coeficiente de } \\
\text { correlação parcial }\end{array}$ & Probabilidade \\
\hline $\mathrm{N}$ o de dentes & $-0,005118$ & 0,937667 \\
Idade & 0,337183 & $0,000000^{*}$ \\
Procedência & 0,127515 & 0,050400 \\
Sexo & 0,114696 & 0,078666 \\
Escolaridade paterna & $-0,013502$ & 0,836528 \\
Escolaridade materna & $-0,017019$ & 0,794799 \\
Renda familiar & $-0,009807$ & 0,880874 \\
O cupação/Maior renda & 0,039779 & 0,543120 \\
Ocupação/Mãe & 0,050895 & 0,436436 \\
Moradia & 0,011895 & 0,855761 \\
Atendimento médico & $-0,068126$ & 0,297299 \\
Visita ao dentista & 0,075295 & 0,249238 \\
Freqüência cons. odont. & $-0,343000$ & $0,000000 *$ \\
Escovação diária & $-0,034013$ & 0,603131 \\
Freqüência escovação & $-0,000929$ & 0,988672 \\
Responsável/Escovação & $-0,086035$ & 0,189971 \\
\hline * p<0,05 & &
\end{tabular}

to estatístico permite analisar diferentes variáveis simultaneamente, onde, em espaço $n$-dimensional, tais variáveis relacionam-se linearmente com o índice ceos. Na Tabela 7, observa-se que, à análise de regressão múltipla, as mesmas variáveis - idade e freqüência de consultas odontológicas - destacam-se por sua significância estatística.

No grupo de Bauru, observou-se redução nos níveis de cárie em relação ao aumento da renda familiar, e no grupo de São Paulo, isso não é tão evidente. Ao teste de correlação parcial e análise de regressão múltipla, essa variável não apresentou significância estatística quanto à influência na experiência de cárie nos grupos avaliados (Tabelas 6 e 7).

As variáveis relativas a educação dos pais são de grande interesse em estudos de saúde infantil, e pesquisas realizadas em vários países demonstram forte associação com a morbimortalidade infantil ${ }^{4,42,43}$ e a utilização de serviços de saúde ${ }^{4}$. Neste estudo, o nível de escolaridade paterna e materna sugeriu apresentar repercussão na prevalência de cárie infantil, porém, quando avaliado no conjunto de outras variáveis, não houve significância estatística (Tabelas 6 e 7).

A análise da utilização dos serviços de saúde permite avaliar a disponibilidade dos serviços e a cobertura de programas preventivos, como pré-natal, imunizações e puericultura. Por outro lado, os dados de utilização permitem fazer inferências sobre a morbidade da população materno-infantil ${ }^{4}$.

A questão do atendimento médico, nos 3 meses anteriores à pesquisa, teve por objetivo coletar da- 
Tabela 7 - Coeficientes de regressão múltipla entre o índice ceos e algumas variáveis socioeconômicas. Análise de regressão múltipla.

\begin{tabular}{|c|c|c|c|}
\hline Variável & Coeficiente de Regr. & Desvio-padrão & Probabilidade \\
\hline $\mathrm{N} \cong$ de dentes & $-0,005381$ & 0,068739 & 0,937668 \\
\hline Idade & 0,399273 & 0,072877 & $0,000000 *$ \\
\hline Procedência & 0,178949 & 0,090991 & 0,050405 \\
\hline Sexo & 0,105645 & 0,059816 & 0,078672 \\
\hline Escolaridade paterna & $-0,016237$ & 0,078605 & 0,836528 \\
\hline Escolaridade materna & $-0,020780$ & 0,079806 & 0,794800 \\
\hline Renda familiar & $-0,008917$ & 0,059437 & 0,880874 \\
\hline O cupação/M aior renda & 0,044457 & 0,073001 & 0,543123 \\
\hline O cupação/Mãe & 0,054972 & 0,070517 & 0,436439 \\
\hline Moradia & 0,010793 & 0,059314 & 0,855762 \\
\hline Atendimento médico & $-0,063221$ & 0,060524 & 0,297304 \\
\hline Visita ao dentista & 0,189709 & 0,164241 & 0,249243 \\
\hline Freqüência cons. odont. & $-0,320547$ & 0,144443 & $0,027434 *$ \\
\hline Escovação diária & $-0,068056$ & 0,130724 & 0,603133 \\
\hline Freqüência escovação & $-0,001778$ & 0,125098 & 0,988672 \\
\hline Responsável/Escovação & $-0,130007$ & 0,098416 & 0,187796 \\
\hline
\end{tabular}

$* p<0,05$

dos sobre a utilização de serviços curativos, oferecendo uma idéia quanto aos padrões de utilização e demanda aos serviços. Esses dados podem também fornecer subsídios ao estudo da morbidade, se levar em consideração outros fatores que determinam essa utilização, como disponibilidade e acessibilidade dos serviços. Com relação à prevalência de cárie nesta amostra, esta variável não apresentou significância estatística.

A questão visita ao cirurgião-dentista insere-se nesse campo, especialmente ao considerar que apenas 5\% da população brasileira têm acesso regular à assistência odontológica, 15\% o fazem irregularmente, ficando o restante sujeito a atendimento eventual ou sem atendimento odontológico algum ${ }^{41}$. Para os entrevistados que relataram resposta positiva a essa questão, foi perguntada a freqüência anual de consultas odontológicas, com objetivo de verificar se tratava de cuidado odontológico eventual ou se sugeria a existência de atendimento odontológico como parte de um programa de saúde, com algum impacto sobre os níveis de saúde bucal das crianças atendidas.

Verificaram-se níveis mais elevados de cárie nas crianças cuja resposta foi positiva à pergunta se faziam visita regular ao dentista. No entanto, essa variável, analisada para o conjunto da amostra estudada, não apresentou significância estatística (Tabelas $6 \mathrm{e}$ 7), mostrando-se a necessidade de outros estudos focalizando esta variável, em amostras com maior número de crianças.

A avaliação da frequiência de consultas odontológicas permite constatar que, entre aqueles que responderam afirmativamente à questão relativa a visita ao dentista, o aumento na freqüência de consultas corresponde à redução da história de cárie de forma estatisticamente significante, ou seja, de alguma forma o cuidado constante repercute na menor frequiência de agravos à saúde bucal, registrada com menores valores ceos. Observou-se um melhor quadro de saúde bucal nas instituições de São Paulo, que provêem cuidados à criança, em proposta de sistema integrado de atenção.

A questão do auto-cuidado em saúde bucal foi abordada através da avaliação da escovação diária e sua freqüência, como parte do cuidado infantil, referido pelos pais/responsáveis. O objetivo dessa abordagem, questionando também se o cuidado era desenvolvido pela própria criança ou por terceiro - adulto, de forma geral -, centrou-se em verificar a existência de alguma preocupação com a saúde bucal, através do desenvolvimento de hábitos de saúde desde a infância ${ }^{15,21,28}$. No conjunto, essas variáveis não apresentaram significância estatística (Tabelas 6 e 7) na influência sobre a freqüência de cárie nesta amostra.

A estimativa dos níveis de associação de variáveis, como escolaridade dos pais, renda, ocupação dos pais, moradia e grupos socioeconômicos com a experiência de cárie na infância, mostra haver um processo em curso na busca de uma explicação para a ampla ocorrência dessa doença entre as populações e a variação no seu padrão. Poucos estudos ${ }^{6,10}$, ${ }^{13,39}$, no entanto, reportam rigor metodológico na caracterização social ou definição de critérios para essa classificação, o que pode dificultar a interpretação dos $\operatorname{achados}^{27}$. 
A identificação de fatores coletivos de risco à cárie dentária, representados pelos condicionantes sociais, econômicos e culturais, surge como forte instrumento para possibilitar à prática odontológica o entendimento do processo saúde-doença em grupos sociais, bem como a identificação precoce de grupos de maior risco para receberem atenção preferencial em programas de saúde.

\section{CONCLUSÕES}

A análise dos resultados encontrados permitiu as seguintes conclusões:

1. O índice ceos verificado nos diferentes grupos etários indica prevalência mais elevada de cárie entre as crianças das creches de Bauru, com diferença estatisticamente significante nas faixas etárias de 3-4 e 5-6 anos.

2. A avaliação simultânea das variáveis sociais sugere a idade e freqüência de consultas odontológicas como fatores com influência estatisticamente significante sobre a prevalência de cárie na amostra estudada.

\section{REFERÊNCIAS BIBLIOGRÁFICAS}

1. ALALUUSUA, S. \& RENKONEN, O. V. Streptococcus mutans establishement and dental caries experience in children from 2 to 4 years old. Scand J. Dent. Res., 91: 4537, 1983 .

2. ANUÁRIO ESTATÍSTICO DO BRASIL: 1992. Rio de Janeiro, Fundação IBGE, 1992.

3. BARROS, B.A. A utilização do conceito de classe social nos estudos de perfis epidemiológicos: uma proposta. Rev. Saúde Pública, 20: 269-76, 1986.

4. BARROS, F.C. \& VICTORA, C.G. Epidemiologia da saúde infantil: um manual para diagnósticos comunitários. São Paulo, HUCITEC-UNICEF, 1991.

5. BEAL, J.F. \& JAMES, P.M.C. Social differences in the dental conditions and dental needs of 5-years-old children in four areas of the West Midlands. Br. Dent. J., 129: 313-8, 1970.

6. BLAIKIE, D.C. Cultural barriers to preventive dentistry. Aust. Dent. J., 24: 398-401, 1979.

7. BOTAZZO, C. Saúde bucal nas práticas coletivas de saúde. São Paulo, 1989.

8. BRASIL. Ministério da Saúde. Divisão Nacional de Saúde Bucal. Levantamento epidemiológico em saúde bucal Brasil, Zona Urbana, 1986. Brasília, Centro de Documentação do Ministério da Saúde, 1988.
3. A baixa eficácia da prevenção em saúde bucal é evidenciada pelo fato de que crianças que já recebem cuidado odontológico mostram tendência a aumento no índice de cárie, ou ainda, que o cuidado odontológico encontra-se, ainda nos dias atuais, centrado no tratamento restaurador.

\section{AGRADECIMENTOS}

Ao Prof. Dr. Ricardo Cordeiro, do Departamento de Saúde Pública da Faculdade de Medicina de Botucatu-UNESP, pela leitura e significativas sugestões. Às diretoras das creches seguintes, pela colaboração: Creche Central e Creche Oeste da COSEASUSP (Coordenadoria de Saúde e Assistência Social da Universidade de São Paulo), e Creche e Centro Educacional Monteiro Lobato, Sociedade Cristã Maria Ribeiro, Creche Madre Clélia, Creche Comunitária Pingo de Gente, Creche e Berçário Nova Esperança, Creche e Berçário Cruzada dos Pastores de Belém, Creche e Berçário São Judas e São Dimas, Berçário Nana Nenê e Escola e Hotelzinho Infantil Menino Jesus, do Município de Bauru.

9. CANTISANO, M.H. et al. Determinação do número de Streptococcus mutans na saliva de crianças com 6 anos de idade e diferentes experiências de cárie. Estomatol. Cult., 13: 44-8, 1983.

10. CARLSSON, P. et al. High prevalence of mutans Streptococci with extremely low prevalence of dental caries. Oral Microbiol. Immunol., 2: 121-4, 1987.

11. CHAVES, M.M. Odontologia social. 3.ed. Rio de Janeiro, Artes Médicas, 1986.

12. DAVENPORT, E.S. Caries in the preschool child: aetiology. J. Dent., 18: 300-3, 1990.

13. FREEMAN, H.E. \& LAMBERT JR., C. Preventive dental behavior of urban mothers. J. Hum. Behav., 6:141-7, 1965.

14. FUKS, A.B. et al. The influence of social and ethnic factors on dental care habits and dental anxiety: a study in Israel. Int. J. Pediatr. Dent., 3: 3-7, 1993.

15. FUNDAÇÃO IBGE. Crianças $e$ adolescentes: indicadores sociais. Rio de Janeiro, 1992.

16. GOLDENBERG, P. Repensando a desnutrição como questão social. Campinas, UNICAMP, 1988.

17. GRAY, M.M. et al. The relationship between caries experience in the deciduous molars at 5 years and in first permanent molars of the same child at 7 years. Community Dent. Health., 8: 3-7, 1991. 
18. GRUEBBEL, A.O. Apud Chaves, M. M. Odontologia social. 3.ed. Rio de Janeiro, Artes Médicas, 1986.

19. HOLM, A. K. Caries in the preschool child: international trends. J. Dent., 18: 291-5, 1990.

20. HOLT, R.D. Caries in the preschool child: British trends. $J$. Dent., 18: 296-9, 1990.

21. HONKALA, E. et al. Familial aggregation of dental health habits in Finland. J. Periodontics, 7: 276-86, 1983.

22. INTERNATIONAL DENTAL FEDERATION. Goals for oral health in the year 2000. Br. Dent. J., 152: 21-3, 1982.

23. JOHNSEN, D.C. et al. Social factors and diet diaries of caries-free and high-caries 2 - to-7-year-olds presenting for dental care in West Virginia. Pediatr. Dent., 2: 279-86, 1980 .

24. KASTE, L.M. et al. The assessment of nursing caries and its relationship to high caries in the permanent dentition. $J$. Public Health Dent., 52: 64-8, 1992.

25. LESER, W. et al. Elementos de epidemiologia geral. Rio de Janeiro, Atheneu, 1988.

26. LEVANTAMENTO epidemiológico básico de saúde bucal: manual de instruções. São Paulo, OMS. 1991.

27. LEVERETT, D.H. \& JONG, A. Variations in use of dental care facilities by low-income white and black populations. $J$. Am. Dent. Assoc., 80: 137-40, 1970.

28. LIMA, J.E. de O. Um plano de prevenção para consultório odontopediátrico. Rev. Gaúcha Odont., 40: 395-9, 1992.

29. LOPES, E.S. \& BASTOS, J.R. de M. O índice CPOD. Bauru, FOB-USP, 1991.

30. METZ, A.S. \& RICHARDS, L.G. Children's preventive dental visits: influencing factors. J. Am. Coll. Dent., 34: $204-$ 12,1967

31. NARVAI, P.C. Saúde bucal: assistência ou atenção? São Paulo, FSP-USP, 1992.

32. PARREIRA, M.L.J. \& MORAES, V.R. de. Relação da prevalência de lesão cariosa nas superfícies mesial dos primeiros molares permanentes e distal dos segundos molares decíduos: estudo comparativo entre escolares de regiões beneficiadas e não pelo fluoreto. Arq. Cent. Est. Cur. Odont., 19: 169-81, 1982.
33. PERSSON, L-A. et al. Nutrition and health in childhood: causal and quantitative interpretations of dental caries. Community Dent. Oral Epidemiol., 12: 390-7, 1984.

34. PINTO, V.G. Saúde bucal: panorama internacional. Brasília, Ministério da Saúde/Secretaria Nacional de Programas Especiais de Saúde/Divisão Nacional de Saúde Bucal, 1990.

35. RAYNER, J.F. Socioeconomic status and factors influencing the dental health practices of mothers. Am. J. Publ. Health., 60: $1250-8,1970$.

36. REED, G.E. \& MCHUGH, R.B. The estimation of dental caries incidence in the presence of diagnostic error. Biometrics, 35: 473-8, 1979.

37. SANTOS, V.I.M. et al. Avaliação da incidência de cárie nos molares permanentes, com o emprego de óxido de zinco e eugenol reforçado, na dentição decídua. Rev. Assoc. Paul. Cirurg. Dent., 38: 33, 1984.

38. SCLAVOS, S. et al. Future caries development in children with nursing bottle caries. J. Pedodont., 13: 1-10, 1988.

39. SILVER, D.H. A comparison of 3-year-olds' caries experience in 1973, 1981 and 1989 in a Hertfordshire town related to family behaviour and social class. Br. Dent. J., 172: 191-7, 1992.

40. STAVENHAGEN, R. Estratificação social e estrutura de classes. In: Velho, O.C. et al. org. Estrutura de classes e estratificação social. 9.ed. Rio de Janeiro, Zahar, 1981. p.133-70.

41. TOLEDO, J.P.G. A saúde bucal no Estado de São Paulo: das ações integradas de saúde ao Sistema Único de Saúde. São Paulo, Organização Panamericana da Saúde/Ministério da Saúde, 1991.

42. VICTORA, C.G. et al. Pobreza e saúde: como medir nível sócio-econômico em estudos epidemiológicos de saúde infantil? In: Congresso Brasileiro de Epidemiologia, $1^{\circ}$, Campinas, 1990. Anais. p. 302-15.

43. WARE, H. Effects of maternal education, women's roles and child care on child mortality. In: Mosley W.H. \& Chen, L.C., ed. Child survival: strategies for research. New York, Population Council, 1984. p.191-214.

44. WINTER, G.B. et al. The prevalence of dental caries in preschool children aged 1-4 years. Br. Dent. J., 1330: 271, 1971.

45. WINTER, G.B. Caries in the preschool child. J. Dent., 18 : 325-6, 1990. 